\title{
Health from the Hive: Potential Uses of Propolis in General Health
}

\author{
Eshwar Shruthi*, B. S. Suma
}

Department of Public Health Dentistry, K.L.E. Institute of Dental Sciences, Bangalore, India.

Email: *drshruthi_80@rediffmail.com

Received January $13^{\text {th }}$, 2012; revised February 20 ${ }^{\text {th }}$, 2012; accepted March 20 ${ }^{\text {th }}, 2012$

\begin{abstract}
The health industry has always used natural products as an alternative, to the conventional allopathic formulations available for the treatment of various afflictions. Propolis, a natural antibiotic is a resinous yellow brown to dark brown substance that honey bees (Apis mellifera) collect from tree buds, sapflows, shrubs or other botanical sources to seal unwanted open spaces in the hive, protecting it from outside contaminants. The main chemical classes present in propolis are flavonoids, phenolics and other various aromatic compounds. Flavonoids are well known plant compounds that have antibacterial, antifungal, antiviral, antioxidant and anti-inflammatory properties. Propolis has been used in general for various purposes and has a promising role in future medicine as well as in dentistry. This paper is an attempt to review various applications of this compound in medicine.
\end{abstract}

Keywords: Propolis; General Health; Safety \& Precautions

\section{Introduction}

There is a great trend nowadays to use natural materials as cure for many diseases. Alternative medicine has made a lot of contributions to modern medical practice [1]. Propolis was used at the time of Egyptian and Greek civilizations which recognized its healing qualities. Hippocrates, the founder of modern medicine, used it for healing sores and ulcers internally and externally. The word Propolis (Russian Penicillin) is derived from the Greek word "pro" before, polis "city" or defender of the city. This non-toxic resinous substance was classified into 12 types according to physicochemical properties and related to geographic locations; however, the botanical origin of only three types were identified (Wander, 1995). A new type of propolis, named Brazilian red Propolis (BRP) because of its color, it has attracted the attention of international business [2]. Propolis is the glue that honey bees (Apis mellifera) use to seal up their hives. Propolis is a sticky filler substance with the aroma of poplar honey and vanilla that is collected by bees from the buds and barks of trees and plants such as horse chest, nuts, poplar and fir trees. The bees take the resin back to their hives and work on it, producing a glue like substance with which they fill cracks, smooth over the interior of the nest, strengthen comb attachments and cover and embalm intruders and other objectionable objects in the hive that are too large to carry out. [1] In nature, or when in

"Corresponding author. room temperature, it is a sticky substance, but becomes hard and brittle at low temperature. It is composed of resin and balsams (50\% - 70\%), essential oils and wax (30\% - 50\%), pollen $(5 \%-10 \%)$ and other constituents which are amino acids, minerals, vitamins A, B complex, $\mathrm{E}$ and the highly active bio-chemical substance known as bioflavonoid (Vitamin P), phenols and aromatic compounds [2]. The main chemical classes present in propolis are flavonoids, phenolics and other various aromatic compounds. Flavonoids and caffeic acid present in propolis are known to play an important role in reducing the inflammatory response by inhibiting lipoxygenase pathway of arachidonic acid. Flavonoids and caffeic acid also aid the immune system by promoting phagocytic activities and stimulates cellular immunity. Propolis is available in the world markets in different forms as capsules, lozenges, tincture and cream in Europe and America. It is already available in Russia as toothpaste. Further, research is being carried out at Oxford University, on the benefits of Propolis [3].

\section{Synonyms}

Apis mellifera L., bee glue, bee propolis, bee putty, Bienenharz (German), Brazilian green propolis, Brazilian propolis, Bulgarian propolis, caffeic acid phenethyl ester (CAPE), cera alba, chizukit, cinnamic acid, flavonoids, galangin, Greek propolis, hive dross, Propolin H, propolis balsam, propolis resin, propolis wax, propolisina (Spanish), 
Russian penicillin, Taiwanese propolis, terpenes, WSDP.

The aim of this review was to draw the attention of dental health care workers to propolis as a natural remedy and its plausible use in dental diseases.

\section{Potential Actions of Propolis (The Use of Propolis in Medicine)}

\subsection{In Medicine}

General medicinal uses of propolis include treatment of the cardiovascular and blood systems (anemia), respiratory apparatus (for various infections), dental care, dermatology (tissue regeneration, ulcers, eczema, wound healingparticularly burn wounds, mycosis, mucous membrane infections and lesions), cancer treatment, immune system support and improvement, digestive tracts (ulcers and infections), liver protection and support and many others [4].

\subsection{Anti-Bacterial Effect}

In a study by Grange and Davey, they used a Propolis dilution of 1:20 in nutrient agar which completely inhibited the growth of $S$. aureaus, S. epidermidis, Enterococcus spp. Corynebacterium spp. B. catarrahlis and B. cereus. This dilution partially inhibited the growth of $P$. aeruginosa and E. coli but had no effect on K. pneumonia. Thus it appeared to have an inhibitory effect on cocci and gram positive rods. Tube dilution studies showed that it was bactericidal for $\mathrm{B}$. cereus and the gram-negative cocci at dilutions of $1: 160$ to $1: 320$, and that growth of the H37RV reference strain of Mycobacterium tuberculosis was totally inhibited at 1:320 and partially inhibited at 1:640 dilution [5].

Koo et al. in Brazil found antibacterial effect of Propolis on S. mutans, S. sanguis and A. naeslundaii in addition to the inhibition of glycosultransferase [6].

\subsection{Anti-Viral Effect}

Serkedjieva conducted an in vitro study on the antiviral activity of six synthetic substances which were esters of substituted cinnamic acids, identical with or analogous to some of the constituent fractions of Propolis. One of them, isopentyl ferculate, inhibited significantly the infectious activity of influenza virus A in vitro and the production of hemalutinins in vivo. In diverse experimental studies, it was found that the maximal inhibition of viral reproduction was observed when test substances were present in the medium during the whole infectious process [7].

\subsection{Anti-Fungal Effect}

Propolis and nine anti-fungal drugs were tested on four fungi that cause infections in humans. It was as effective as (or more effective than) some of the other preparations against three of the fungi, and in some tests, its activity was enhanced in the presence of propylene glycol. Propolis and propylene glycol gave better results against Scopulariopsis breveicaulis than any of the drugs tested [8].

\subsection{Anti-Oxidant Effect}

Krol et al. described the remarkable medical property of the ethanolic extract of Propolis (EEP), that is the protection against gamma radiation. They performed their experiment on mice and found that the anti-oxidative effect could be attributed to its radical scavenging ability. They also demonstrated the ability of increasing amounts of EEP to inhibit luminol H202 chemiluminescence in vitro, and suggested that its anti-oxidative capacity was partly due to its high content of flavenoids [9].

An Italian Study investigated the antioxidant activity of a propolis extract deprived of caffeic acid phenethyl ester (CAPE). Propolis extract (with and without CAPE) and its active components showed a dose-dependent free radical scavenging effect, a significant inhibition of xanthine oxidase activity, and an antilipoperoxidative capacity. Propolis extract with CAPE was more active than propolis extract without CAPE. The experimental evidence, therefore, suggests that CAPE plays an important role in the antioxidant activity of propolis [10].

\subsection{Protective Action of Propolis on Cartilage}

An Italian study states that this natural compound and its active principle, caffeic acid phenethyl ester (CAPE), were able to contrast the harmful effects of IL-1beta.Our data clearly demonstrated the protective action of propolis in cartilage alteration, that appears greater than that elicited by indomethacin, commonly employed in joint diseases [11].

\subsection{In Vaccines}

To be effective, most vaccines typically depend on the inclusion of substances known as adjuvants that stimulate an immune response. Researchers seeking to develop a vaccine against Suid herpesvirus type1 (SuHV-1), which causes an infectious disease among swine, combined green propolis with an ethanol extract and tested it on mice. The mice showed an increased cellular immune response and increased protection against SuHV-1. This response did not occur with propolis alone, but only when the antigen was absorbed in a particulate adjuvant, like aluminum hydroxide. Thus, when associated with auxiliary substances like aluminum hydroxide, green propolis extract may increase the potency of vaccines, especially those that depend on the cellular immune response for protection [12]. 


\subsection{Effect on Cancer}

Scheller demonstrated the anti-tumoral effect in mature mice bearing Ehrlich carcinoma. Survival rate after EEP treatment was compared to that of bleomycin, given alone or in combination every 2 days for thirty-six days and followed up for fourteen additional days. The survival rate at fifty days was 55\% after EEP and $40 \%$ after bleomycin, while all the mice treated with EEP plus bleomycine combination demonstrated shorter survival than the controls. It was concluded that while the in vivo activity of bleomycin was reduced in the presence of cytochrome-c-reductase inhibitors (as some of the EEP components) are the anti-tumoral property of EEP in the experimental animal model studied was significant and lasting [13].

A literature review finds that propolis's pharmacological properties make it safe and effective as an adjunct for patients receiving cancer treatment:

- Biological therapy. Biological therapy works hand in hand with the immune system. Propolis's biological activities such as antitumoral activity, DNA protection, free-radicals scavenging, and immune stimulation act in synergy with each other and with conventional chemotherapy medication.

- Synergy with chemotherapy. Antioxidants may boost the effects of anti-carcinogenic drugs, thus enabling a decrease in the administered dose and in turn leading to a reduction in side effects. They may also influence the response to chemotherapy.

- Anti-inflammatory activity. This results from propolis's inhibitory effect on prostaglandins, leukotrienes, and histamine release.

- Healing activity. Propolis promotes epithelial formation as well as vascular and fibroblastic neoformation of the connective tissue.

- Antimicrobial activity. Propolis' flavonoids and phenolic acids are pharmacologically active compounds that have effects on bacteria, fungi, and viruses [14].

\subsection{Effect on Upper Respiratory Infections}

German study investigated the bactericidal effect against several strains isolated from patients with infections in their upper respiratory tracts [15].

\subsection{In Cosmetics}

Dermatological and cosmetic applications are at this time probably the most common uses for propolis and its extracts. Its effects on tissue regeneration and renovation have been well studied. Together with its bactericidal and fungicidal characteristics it provides many benefits in various applications in cosmetics [4].

\section{Safety \& Precautions}

\subsection{Bee Propolis-How Safe Is It?}

In general, propolis is safe. It is a non toxic substance and for most people, will not caused irritation when used as supplements or applied to skin. However, like other honey bee products, there are people who are allergic to propolis. Allergic reaction due to this substance was first reported in beekeepers as an occupational effect but is now seen mainly in individuals who use propolis in cosmetics and supplement to treat various health conditions. It is believed that a substance called caffeic acids to be one of the causes of allergies to propolis.

\subsection{Who Can Be Allergic to Propolis?}

- Those allergic to pollen;

- Asthma patients;

- Allergic to bee stings;

- Pregnant women.

There have not been many published clinical trials on the effect of propolis on pregnant women. Because the information in this area is limited, it has been advised to avoid using propolis during pregnancy and breastfeeding.

\subsection{The Symptoms of Allergies}

If allergic to propolis, it may cause redness of skin, develop rashes, swelling, itching, fluid collection, fever and may even lead skin to crack (including a severe allergic reaction called anaphylaxis). Apart from that, it may also irritate the skin area where it is applied on, cause eczema, lesions, psoriasis or mouth sores [16].

\subsection{Interactions with Drugs}

Many tinctures contain high levels of alcohol and may cause nausea or vomiting when taken with metronidazole or disulfiram. Propolis may produce additive effects when taken with antimicrobial drugs.

Propolis may interact with the following: anticoagulants, H. pylori agents, antibiotics, anti-cancer agents (antineoplastics), antifungals, anti-inflammatories, infertility agents, anti-HIV agents (antiretrovirals), immunosuppressants, and osteoporosis agents.

\subsection{Interactions with Herbs and Dietary Supplements}

Balsam of Peru and propolis are both known to cause allergic sensitization in some people and have multiple compounds in common, such as benzyl benzoate, benzyl cinnamate, benzyl alcohol, benzoic acid, cinnamic acid, caffeic acid, cinnamic alcohol, and vinallin. An increased risk of allergic sensitization may occur if both products are used together [17]. 


\section{Summary and Conclusion}

The evidence on bee propolis is very promising. Not only can it be applied topically it may also be considered as an alternative to antibacterial, antiviral, antifungal, anti inflammatory, antibiotic, antioxidant property in treatment of low grade infections. Allergy and sensitivity to propolis is uncommon but patients should be asked about adverse reactions to bee stings, allergy to bee products and sensitivity to pollen. In conclusion, propolis is a natural medication with a promising future but further studies should be conducted to investigate its merit and demerits in clinical medicine.

\section{Recommendations}

- Studies should be conducted on the effects of propolis and these studies should meet the requirements of basic principles of clinical research and trial.

- Both clinical trials and demonstration studies should be carried out to determine the efficacy and effectiveness of propolis in human subjects.

- The primary health care notion of appropriate technology should be encouraged through.

- Controlled trials of native natural herbal products as alternative medicine for good general and dental health of the community.

\section{REFERENCES}

[1] K. Almas, A. Dahlan and A. Mahmoud, "Propolis as a Natural Remedy: An Update,” Saudi Dental Society, Vol. 13, No. 1, 2001, pp. 45-49.

[2] A. Parolia, M. S. Thomas, M. Kundabala and M. Mohan, "Propolis and Its Potential Uses in Oral Health," International Journal of Medicine and Medical Sciences, Vol. 2, No. 7, 2010, pp. 210-215.

[3] C. Scully, "Propolis: A Background,” British Dental Journal, Vol. 200, No. 7, 2006, pp. 359-360. doi:10.1038/sj.bdj.4813435

[4] Bee Propolis and Its Medicinal Uses, 3 December 2005. http://www.pharmainfo.net/reviews/bee-propolis-and-itsmedicinal-uses

[5] J. M. Grange and R. W. Davey, "Anti-Bacterial Propertiesof Propolis,” Journal of the Royal Society of Medicine, Vol. 83, No. 3, 1990, pp. 159-160.

[6] H. Koo, J. A. Cury, P. L. Rosaleu and Y. K. Park, "Effects of Propolis from Two Different Regions of Brazil on Oral
Microorganisms,” Journal of Dental Research, Vol. 77, 1998, p. 1157.

[7] J. Serkedjieva, “Anti-Influenza Virus Effect of Some Propolis Constituents and Their Analogues (Esters of Substituted Cinnamic Acids)," Journal of Nat Products, Vol. 55, No. 3, 1992, pp. 294-302. doi:10.1021/np50081a003

[8] J. Miller-Clere, D. Michel, J. Simeray and J. P. Chaumon, "Preliminary Study of the Anti-Fungal Properties of Propolis Compared with Some Commercial Products,” Fac Medicine \& Pharmacy, Besancon Monograph Cedex, France 1987.

[9] W. Krol, Z. Czuba, S. Scheller, J. Gabrys, S. Grabiec and J. Shani, "Anti-Oxidant Property of Ethanolic Extract of Propolis (EEP) as Evaluated by Inhibiting the Chemilminescence Oxidation of Luminal,” Biochemistry International, Vol. 21, No. 4, 1990, pp. 593-597.

[10] A. Russo, R. Longo and A. Vanella, “Antioxidant Activity of Propolis: Role of Caffeic Acid Phenethyl Ester and Galangin,” Fitoterapia, Vol. 73, Suppl. 1, 2002, pp. S21S29. doi:10.1016/S0367-326X(02)00187-9

[11] V. Cardile, A. Panico, B. Gentile, F. Borrelli and A. Russo, "Effect of Propolis on Human Cartilage and Chondrocytes,” Life Science, Vol. 73, No. 8, 2003, pp. 1027-1035. doi:10.1016/S0024-3205(03)00381-3

[12] G. Fischer, et al., "Immunodomodulation Produced by a Green Propolis Extract on Humoral and Cellular Responses of Mice Immunized with SuHV-1," Vaccine, Vol. 25, No. 7, 2007, pp. 1250-1256. doi:10.1016/j.vaccine.2006.10.005

[13] S. Scheller, W. Krol, J. Swiacik, K. S. Owcare, J. Gabrys and J. Shani, "Antitumocal Property of Ethanolic Extract of Propolis in Mice-Bearing Ehrlich Carcinoma, as Compared to Bleomycin,” Department of Microbiology, Silesian School of Medicine, Zabrze-Rokitnica, Monograph Poland, 1989.

[14] J. Galvao, et al., "Biological Therapy Using Propolis as Nutritional Supplement in Cancer Treatment,” International Journal of Cancer Research, Vol. 3, No. 1, 2007, pp. 43-53. doi:10.3923/ijcr.2007.43.53

[15] J. Focht, S. H. Hansen, J. V. Nielsen, A. van den BergSegers and R. Riezler, "Bactericidal Effect of Propolis in Vitro against Agents Causing Upper Respiratory Tract Infections,” Bioscientia, Vol. 43, No. 8, 1993, pp. 921-923.

[16] P. Wander, “Taking the Sting out of Dentistry,” Dental Practice, Vol. 25, 1995, pp. 3-4.

[17] US National Library of Medicine and National Institutes of Health, "Propolis: Medlineplus Supplements,” NLM \& NIH, Bethesda. 